\title{
INTRUSION CONFINEMENT BY ISOLATION IN INFORMATION SYSTEMS
}

\author{
Peng Liu, Sushil Jajodia and Catherine D. McCollum
}

\begin{abstract}
System protection mechanisms such as access controls can be fooled by authorized but malicious users, masqueraders, and misfeasors. Intrusion detection techniques are therefore used to supplement them. However, damage could have occurred before an intrusion is detected. In many computing systems the requirement for a high degree of soundness of intrusion reporting can yield poor performance in detecting intrusions and can cause long detection latency. As a result, serious damage can be caused either because many intrusions are never detected or because the average detection latency is too long. The process of bounding the damage caused by intrusions during the process of intrusion detection is referred to as intrusion confinement. We justify the necessity for intrusion confinement during detection by a probabilistic analysis model, and propose a general solution to achieve intrusion confinement. The crux of the solution is to isolate likely suspicious actions before a definite determination of intrusion is reported. We also present a concrete isolation protocol in the file system context to evaluate the feasibility of the general solution, which can be applied in many types of information systems.
\end{abstract}

Keywords: Intrusion confinement, intrusion detection, isolation, profile-based detection, signature-based detection

\section{INTRODUCTION}

Recently there has been increasing emphasis on supplementing protection of networks and information systems with intrusion detection $[10,14,11]$ and numerous intrusion detection products have emerged commercially. Recognizing that access controls, filtering, and other protection mechanisms can be defeated or bypassed by would-be attackers who take advantage of remaining vulnerabilities, intrusion detection systems monitor system or network activity to discover attempts to disrupt or gain illicit access to systems. Intrusion detection must be concerned both with attempts by external penetrators to enter 
or interfere with the system and by authorized users to exceed their legitimate access or abuse the system in some way. The latter case also includes seemingly authorized users, such as masqueraders operating under another user's identification (id) and password, or outside attackers who successfully gained system access but eluded detection of the method of entry. The methodology of intrusion detection can be roughly classed as being either based on statistical profiles or on known patterns of attacks, called signatures.

Statistical profile-based systems compare relevant data by statistical or other methods to representative profiles of normal, expected activity on the system or network. Deviations indicate suspicious behavior. In these systems, there are stringent requirements on not only reporting an intrusion accurately (this is necessary because abnormal behavior is not always an intrusion) but also detecting as many intrusions as possible (usually, not all intrusions can be detected). However, these two requirements can often result in conflicting design goals.

Based on the assumption that the more significant the deviation, the larger the possibility that the behavior of a user is an intrusion, in order to ensure a high degree of soundness of intrusion reporting, significant anomaly is required to raise a warning. However, doing it this way usually decreases the number of intrusions that can be detected because intrusions characterized by gradual anomaly can be overlooked by the detector (a formal analysis is presented in Section 2), in which case many intruders may stay at large and cause substantial damage. Moreover, when the anomaly of an intrusion is accumulated slowly, detecting it can still cause a long latency even if it is characterized by significant anomaly. As a result, substantial damage can be caused by an intruder within the latency. Relaxing the significancy requirement on deviations can mitigate these problems; however, the soundness of intrusion reporting can be dramatically degraded. In other words, innocent users may be mistaken for malicious ones and legal service requests may be denied in many situations. This is because trustworthy users may gradually change their behavior in nonsignificant ways.

With the requirement of a given degree of soundness, the process of bounding the damage caused by undetected and/or detected intrusions during their latencies is referred to as intrusion confinement. Intrusion confinement also encompasses corresponding strategies and mechanisms taken to enable the process.

Signature-based detection examines sniffer logs, audit data, or other data sources for evidence of operations, sequences, or techniques known to be used in particular types of attacks. Signature-based detection techniques cannot be used to detect new, unanticipated patterns that could be detected by statistical profile-based detection techniques. However, they are used to detect known attacks. Although a behavior that partially matches a signature will not cause 
damage (see that the behavior is not an intrusion because otherwise the signature can be shorter), serious damage may have already been caused by the time a signature is matched. The process of bounding this kind of damage is also referred to as intrusion confinement.

This paper makes two contributions: (1) It gives a simple probabilistic model to measure the effectiveness of an intrusion detection system and to justify the necessity of intrusion confinement. Based on the degree of effectiveness, the system security officer (SSO) can decide whether to enforce intrusion confinement and, if so, what mechanisms and strategies to apply for intrusion confinement. (2) It gives a general solution of intrusion confinement that is closely coupled with intrusion detection mechanisms and can be applied in many kinds of information systems. The basic idea is to isolate suspicious actions before an intrusion is reported. The solution is illustrated in the context of a file system.

This work has relevance to information warfare (IW) defense $[2,3,12,13$, 15]. As pointed out by Ammann, et al. [2], information warfare defense does everything possible to prevent attacks from succeeding, but it also recognizes that attempting to prevent information attack is insufficient. Attacks that are successful to some degree must be recognized as unavoidable, and comprehensive support for identifying and responding to attacks is required.

The rest of the paper is organized as follows. In Sections 2 and 2.3, we use a probabilistic model to justify the necessity of intrusion confinement. Section 3 presents a general solution of intrusion confinement. In Section 4, we evaluate the feasibility of our solution by presenting a concrete isolation protocol which is applied to a file system. In Section 5, we conclude the paper.

\section{WHY APPLY INTRUSION CONFINEMENT}

Informally, suspicious behavior is the behavior that may have already caused some damage, or may cause some damage later on, but was not reported as an intrusion when it happened. In our model, suspicious behavior emerges in four situations: (1) In statistical profile-based detection: (a) In order to get a high degree of soundness of intrusion reporting, some intrusions characterized by gradual deviations may stay undetected. The corresponding behaviors can be reported as suspicious. (b) For a detection with a long latency, the corresponding behavior can be reported as suspicious in the middle of the latency. (c) Legitimate behavior can be reported as suspicious if it is sufficiently unlike the corresponding profile. (2) In signature-based detection, partial matching of a signature can trigger a report of suspicious behavior.

In the remainder of this section, we present a probabilistic model for justifying the necessity of intrusion confinement. Note that all of the statistics used in 
this section were computed based on the audit trail, where the entire intrusion history is assumed to be recorded.

\subsection{FOR PROFILE-BASED DETECTION}

Consider a statistical profile-based detection system where a user $U_{i}$ accesses the system through sessions. A session of $U_{i}$ begins when $U_{i}$ logs in and ends when $U_{i}$ logs out. A behavior of $U_{i}$ is a sequence of actions that can last across the boundaries of sessions. A short-term behavior of $U_{i}$ is a behavior that is composed of a sequence of $U_{i}$ 's most recent actions. In contrast, a long-term behavior of $U_{i}$ is also a sequence of $U_{i}$ 's most recent actions but it is much longer than the short-term behavior. We assume the intrusion detector is triggered in every $m$ actions (or $m$ audit records), that is, after $m$ new actions are executed, both the current short-term behavior and long-term behavior of $U_{i}$ will be upgraded and the deviation of the new short-term behavior from the new long-term behavior will be computed. When a short-term behavior is upgraded, its oldest $m$ actions will be discarded and the newest $m$ actions will be added.

In the following presentation, we use "behavior" to denote a short-term behavior for brevity. The access history of $U_{i}$ can be specified as a sequence of behaviors, although two behaviors in the sequence can have multiple actions in common. We model the access history of $U_{i}$ as a discrete stochastic process $x(t)$ where $x\left(t_{i}\right)$, which indicates the type of the behavior of $U_{i}$ at time $t_{i}$, can take one of three possible values, namely, LEGITIMATE, SUSPICIOUS, and INTRUSION, usually with different probabilities. It should be noted that $x(t)$ is theoretically not an independent process because: (1) the behavior of $U_{i}$ at time $t_{i-1}$ is a part of the long-term behavior that is the profile of $U_{i}$ at time $t_{i}$, hence $x(t)$ is affected by $x(t-1)$; (2) the behavior of $U_{i}$ at times $t_{i-1}$ and $t_{i}$ can share multiple actions; (3) the behavior of $U_{i}$ at times $t_{i-1}$ and $t_{i}$ can have semantic relationships. For example, a LATEX command is usually followed by a DVIPS command when a TEX file is compiled. However, it is reasonable to simplify the model by viewing $x(t)$ as an independent process in many situations when the profile covers a relatively long long-term behavior and when the overlap of neighbored behaviors is only a small part of each behavior because at this point the effect of the behavior of $U_{i}$ at time $t_{i-1}$ on the profile is too weak to affect $x(t)$, and neighbored behaviors can be viewed as having no overlaps. For simplicity, we model $x(t)$ in the following presentation as an independent discrete stochastic process. In addition, we assume that the access histories of any two users $U_{i}$ and $U_{j}$ are independent of each other. Note that our goal is showing that intrusion confinement is necessary, as opposed to evaluating accurately the performance of intrusion detection systems. 
We assume that statistical methods, such as the methods proposed in NIDES [7], are used in the system. We assume that both the short-term and longterm behaviors of $U_{i}$ at time $t$ are described by a vector of $n$ intrusion detection measures (or variables). We denote them as $\overrightarrow{v_{s}}=\left(v_{s 1}, \ldots, v_{s n}\right)$ and $\overrightarrow{v_{l}}=\left(v_{l 1}, \ldots, v_{l n}\right)$, respectively. The deviation of $\overrightarrow{v_{s}}$ from $\overrightarrow{v_{l}}$ is specified by the distance, denoted $d\left(\overrightarrow{v_{s}}, \overrightarrow{v_{l}}\right)$, from the point defined by $\overrightarrow{v_{s}}$ to the point defined by $\overrightarrow{v_{l}}$ in the $n$-dimension space. A warning is raised if $d\left(\overrightarrow{v_{s}}, \overrightarrow{v_{l}}\right)$ is sufficiently long.

We evaluate the effectiveness of such an statistical profile-based detection system with two measures: (1) The rate of detection, denoted $R_{d}$, is the percentage of the intrusions that can be detected among all of the intrusions. $R_{d}$ indicates the general ability of a detector in detecting intrusions. (2) The rate of errors, denoted $R_{e}$, is the probability that a reported intrusion is actually not an intrusion. $R_{e}$ indicates the soundness of a detector. The smaller $R_{e}$ is, the higher degree of soundness is achieved.

$R_{d}$ and $R_{e}$ can be formalized based on the following seven parameters: (1) $D_{i}$, the value of distance such that if $d\left(\overrightarrow{v_{s}}, \overrightarrow{v_{l}}\right) \geq D_{i}$, then $\overrightarrow{v_{s}}$ is reported as an intrusion. (2) $P_{i}$, the probability that when a behavior is reported as an intrusion, that is, $d\left(\overrightarrow{v_{s}}, \overrightarrow{v_{l}}\right) \geq D_{i}$, it is really an intrusion. (3) $D_{s}$, the value of distance such that if $d\left(\overrightarrow{v_{s}}, \overrightarrow{v_{l}}\right) \geq D_{s}$, then $\overrightarrow{v_{s}}$ is reported as suspicious. (4) $P_{s}$, the probability that when a behavior is reported as suspicious, that is, $d\left(\overrightarrow{v_{s}}, \overrightarrow{v_{l}}\right) \geq D_{s}$, it is an intrusion. (5) $A_{i}$, the probability that a behavior deviates from the corresponding long-term behavior with $d\left(\vec{v}_{s}, \vec{v}_{l}\right) \geq D_{i}$. (6) $A_{s}$, the probability that a behavior deviates from the corresponding long-term behavior with $d\left(\overrightarrow{v_{s}}, \overrightarrow{v_{l}}\right) \geq D_{s}$. (7) $P_{g}$, the probability that a behavior with $d\left(\overrightarrow{v_{s}}, \overrightarrow{v_{l}}\right) \leq D_{s}$ is an intrusion. We introduce $D_{s}, P_{s}$, and $A_{s}$ to model intrusion confinement. Note that $D_{s}$ is less than $D_{i}$; hence, a reported intrusion must be suspicious.

$$
\begin{aligned}
& R_{d}=\frac{A_{i} P_{i}}{A_{s} P_{s}+\left(1-A_{s}\right) P_{g}} \\
& R_{e}=1-P_{i}
\end{aligned}
$$

Example 1 Assume the SSO decides that $P_{i}$ should be at least 0.90 to ensure a high degree of soundness. As a result, the corresponding $D_{i}$ can be determined to ensure such a $P_{i}$. However, if we consider the situation where $A_{i}=0.01$, $A_{s}=0.10, P_{s}=0.50$, and $P_{g}=0.002$, then the rate of detection $R_{d}=0.174$, which is very low. On the other hand, if we decrease the value of $P_{i}$ to detect more intrusions, for example, we set the value of $D_{i}$ to that of $D_{s}$, then the rate of error $R_{e}=1-P_{s}=0.50$, which is too high.

The above example shows that in many situations if we want to achieve a low rate of errors, then we cannot achieve a high rate of detection. Therefore, the two requirements can often result in conflicting design goals. Since $R_{e}$ cannot be very high because otherwise substantial legitimate service requests 
will be denied, a high rate of detection cannot be achieved in many cases. Thus, many intrusions can stay undetected (in Example 1, the undetected intrusion rate is $82.6 \%$ ), and the latency of an intrusion can be very long. As a result, serious damage can be caused.

Intrusion confinement can bound this damage. The set of actions that should be isolated can be specified as follows. Note that since $D_{s}$ is determined by the SSO based on the value of $P_{s}$ he prefers, intrusion confinement systems can be flexibly configured.

Definition 1 A short-term behavior described by $\overrightarrow{v_{s}}$ is suspicious if $d\left(\vec{v}_{s}, \overrightarrow{v_{l}}\right) \geq$ $D_{s}$. Here, $D_{s}$ is determined by the value of $P_{s}$, which is chosen by the SSO.

By isolating suspicious behaviors, we can often protect the system from the damage caused by most of the intrusions. The effectiveness of isolation can be measured by the rate of isolation, denoted $R_{i}$, which is the percentage of the intrusions that will be isolated among all of the intrusions. $R_{i}$ is formalized as follows:

$$
R_{i}=\frac{A_{s} P_{s}}{A_{s} P_{s}+\left(1-A_{s}\right) P_{g}}
$$

In Example 1, if we keep $P_{i}$ at $0.90, R_{i}$ is $96.5 \%$.

\subsection{FOR SIGNATURE-BASED DETECTION}

We specify a signature as a sequence of events leading from an initial limited access state to a final compromised state $[17,4,5,18,19,8]$. Each event causes a state transition from one state to another state. We identify a signature with length $n$, denoted $\operatorname{Sig}(n)$, as $\operatorname{Sig}(n)=s_{0} E_{1} s_{1} \ldots E_{n} s_{n}$, where $E_{i}$ is an event and $s_{i}$ is a state, and $E_{i}$ causes the state transition from $s_{i-1}$ to $s_{i}$. For simplicity, intra-event conditions are not explicitly shown in $\operatorname{Sig}(n)$, although they are usually part of a signature.

A partial matching of a signature $\operatorname{Sig}(n)$ is a sequence of events that matches a prefix of $\operatorname{Sig}(n)$. A partial matching is not an intrusion; however, it can predict that an intrusion specified by $\operatorname{Sig}(n)$ may occur. The accuracy of the prediction of a partial matching, denoted $s_{0} E_{1} s_{1} \ldots E_{m} s_{m}$, can be measured by the following parameter: $\mathbf{P}_{\mathbf{m}}$, the probability that the partial matching can lead to an intrusion later. Assume the number of the behaviors that match the prefix is $N_{p}$ and the number of the intrusions that match the prefix is $N_{i}$, then $P_{m}=N_{i} / N_{p}$.

In signature-based detection, the set of actions that should be isolated is defined as follows. Isolating suspicious behavior can surely confine damage in signature-based detection because the behavior that is actually an intrusion will, with a high probability, be prevented from doing harm to the system. 
Definition 2 In signature-based detection, a behavior is suspicious if it matches the prefix of a signature but not the whole signature, and $P_{m}$ of the prefix is greater than or equal to a threshold that is determined by the SSO.

\subsection{WHEN TO APPLY INTRUSION CONFINEMENT}

In signature-based detection, the decision of whether to enforce intrusion confinement on a known attack that is specified by a signature is dependent on the seriousness of the damage that will be caused by the attack and the value of $P_{m}$ for each prefix of the signature. For example, if the damage is strongly undesirable, and there exists a prefix whose $P_{m}$ is sufficiently large, then intrusion confinement can be enforced by isolating the behavior that matches the prefix.

In statistical profile-based detection, however, it can be tricky to make the decision. As shown in Section 2, since degrading the requirement on $R_{e}$ usually can improve $R_{d}$, the SSO may want to find a trade-off between $R_{e}$ and $R_{d}$; thus, the cost of isolation would be avoided. However, a satisfactory trade-off may not be achievable in some systems since the relationship between $R_{e}$ and $R_{d}$ can dramatically differ from one system to another.

Consider two systems with the same set of parameters and the same associated values as in Example 1. When $P_{i}$ is degraded from 0.90 to 0.85 in both of the systems, the other parameters may take the following values: (a) For system $1, P_{i}=0.85, P_{s}=0.50, A_{i}=0.05, A_{s}=0.10, P_{g}=0.002$. (b) For system $2, P_{i}=0.85, P_{s}=0.50, A_{i}=0.02, A_{s}=0.10, P_{g}=0.002$. It is easy to get the following result: In system $1, R_{d}=0.82$ and $R_{e}=0.15$; In system 2, $R_{d}=0.33$ and $R_{e}=0.15$. It is clear that when $P_{i}$ is degraded to 0.85 , system 1 performs much better than system 2 . At a result, the SSO may need only to enforce intrusion confinement in system 2.

\section{HOW TO APPLY INTRUSION CONFINEMENT 3.1 ARCHITECTURE SUPPORT}

The architecture of an intrusion confinement system from the perspective of information warfare [2] is shown in Figure 5.1.

The Policy Enforcement Manager enforces the access controls in accordance with the system security policy on every access request. We assume no data access can bypass it. We further assume that users' accesses will be audited in the audit trail.

The Intrusion Detection and Confinement Manager applies either statistical profile-based detection techniques or signature-based detection techniques, or both to identify suspicious behavior as well as intrusions. The detection is typically processed based on the information provided by the audit trail. 


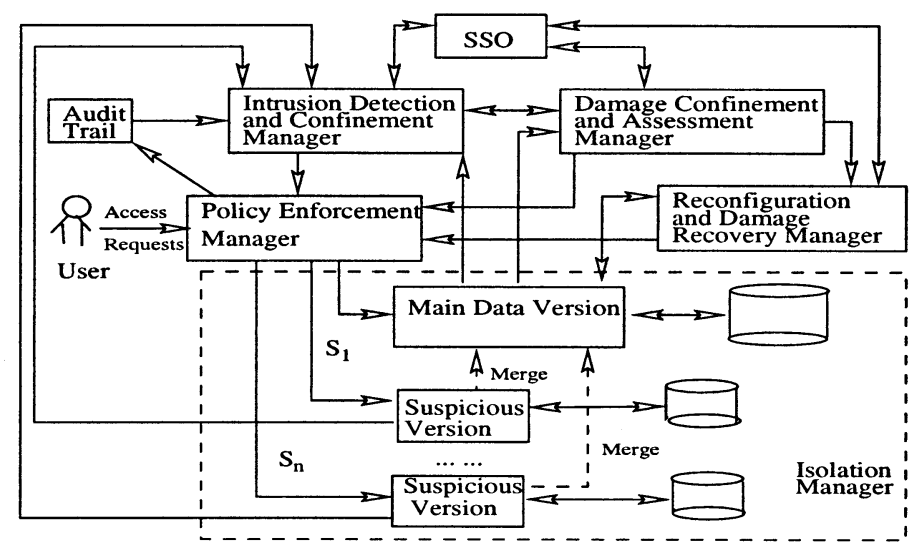

Figure 1.1 Architecture of the intrusion confinement system.

When a suspicious behavior is detected, the corresponding user is marked suspicious. At this point, first we need to deal with the effects that the user has already made on the Main Data Version because these effects may have already caused some damage. In signature-based detection systems, we can accept these effects because a partial matching is not an intrusion. In statistical profile-based detection systems, if the SSO does not think these effects can cause any serious damage, we can accept these effects; if the SSO thinks these effects can cause intolerable damage, we can isolate and move these effects from the main data version to a separate Suspicious Data Version, which is created to isolate the user. The process of isolation may need to roll back some trustworthy actions that are dependent on the suspicious actions. At this point, we can apply another strategy that moves the effects of these suspicious actions as well as the affected trustworthy actions to the suspicious data version.

Second, the Intrusion Detection and Confinement Manager notifies the Policy Enforcement Manager to direct the subsequent suspicious actions of the user to the separate data version. Since we focus on the isolation itself, we can simply assume that when a suspicious behavior starts to be isolated, no damage has been caused by the behavior. Note that there can be several different suspicious users, e.g., $S_{1}, \ldots, S_{n}$, being isolated at the same time. Therefore, multiple suspicious data versions can exist at the same time.

When a suspicious user turns out to be malicious, that is, his/her behavior has led to an intrusion, the corresponding suspicious data version can be discarded to protect the main data version from harm. On the other hand, when the user turns out to be innocent, the corresponding suspicious data version is merged into the main data version. A suspicious behavior can be malicious in several ways: (1) In signature-based detection, a complete matching 
can change a suspicious behavior to malicious. (2) Some statistics of gradual anomaly, such as frequency and total number, can make the SSO believe that a suspicious behavior is malicious. (3) The SSO can find that a suspicious behavior is malicious based on some non-technical evidences. A suspicious behavior can be innocent in several ways: (1) In signature-based detection, when no signatures can be matched, the behavior proves innocent. (2) The SSO can prove it to be innocent by some non-technical evidence. For example, the SSO can investigate the user directly. (3) Some statistics of gradual anomaly can also make the SSO believe that a behavior is innocent.

Since intrusion confinement cannot isolate every intrusion in most cases (The rate of isolation, $R_{i}$, is usually less than 1), intrusions do happen on the main data version. When such an intrusion is detected (this type of intrusion is usually detected by some other approaches beyond the standard mechanisms), the corresponding user is marked as malicious. The Intrusion Detection and Confinement Manager then notifies the Damage Confinement and Assessment Manager to confine and assess the damage caused by the intrusion. The confinement is done by notifying the Policy Enforcement Manager to restrict the access by the user by either rejecting his/her further access or isolating the damage to prevent further spread. Concrete damage confinement mechanisms are out of the scope of the paper.

After the damage is assessed, the Reconfiguration Manager reconfigures the system to allow access to continue in a degraded mode while repair is being done by the Damage Recovery Manager. In many situations, damage assessment and recovery are coupled with each other closely. For example, recovery from damage can occur during the process of identifying and assessing damage. Also, the system can be continuously reconfigured to reject accesses to newly identified, damaged data objects and to allow access to newly recovered data objects. Interested readers can refer to [1,9] for more details on damage confinement, damage assessment, system reconfiguration, and damage recovery mechanisms in the database context.

\subsection{SYSTEM CHARACTERISTICS}

When a suspicious user $S_{i}$ is detected, there are several different types of data flow between the trustworthy actions working on the main data version and the suspicious actions of $S_{i}$ : (a) Complete Isolation: Updates by $S_{i}$ 's actions are not disclosed to any trustworthy action, and any updates by a trustworthy action are not disclosed to $S_{i}$. (b) One-way Isolation: Updates by $S_{i}$ 's actions are not disclosed to any trustworthy action, but all updates by a trustworthy action are disclosed to $S_{i}$. When an update is disclosed to an action, the action can read the updated value. (c) Partial Isolation: Some updates by $S_{i}$ 's actions are disclosed to trustworthy actions, and vice versa. This can happen 
when $S_{i}$ 's updates on some data objects are not considered risky, while anything else $S_{i}$ attempts to update is confined to the suspicious version. By partial isolation, if some of the updates by trustworthy users are considered sensitive, they could be selectively withheld from being propagated to the suspicious version where they would be divulged to the suspicious user. One drawback of disclosing $S_{i}$ 's updates to trustworthy users is that after $S_{i}$ is revealed as malicious, some trustworthy actions affected by $S_{i}$ 's updates may have to be backed out.

Data flows among suspicious users can also be grouped into the above three types. However, we have not found many situations where it is useful to disclose updates among suspicious versions, except when the SSO finds that several suspicious users cooperate with each other to do something. At this point, it is helpful that these suspicious users are isolated within one suspicious version because if they are malicious users who collude to do intrusions and to protect themselves from being detected, then isolating each user within a separate version can alert the user to the fact that something is wrong. If they are innocent users, then isolating each user in a separate version can prevent $\mathrm{him} /$ her from inter-communicating. However, since collusions are usually difficult to detect, and it is usually a complicated and computing intensive process to ensure the correctness of such data flows. We will not address the problem in this paper and we would like to address it in our future research.

When a user is discovered to be malicious or innocent, a decision must be made on how to accept and merge his/her updates into the main data version. At this point, two types of mergers are possible: (a) Complete Merger: When a user is discovered to be malicious, all of his/her updates are discarded. When a user is discovered to be innocent, all of his/her updates are considered for merging. (b) Partial Merger: Remerging of the data versions could be partial. Even if the user were found to be a malefactor, it might be desirable to accept certain of his/her updates into the main database version rather than discarding them all outright.

Because suspicious action histories keep growing, conflicts may arise between trustworthy and suspicious actions. As a result, the main data version and these suspicious data versions may become inconsistent. For example, a trustworthy action and a suspicious action may update the same data object $x$; thus, neither the main data version of $x$ nor the suspicious data version of $x$ is correct when we decide to merge the suspicious version into the main version.

The techniques to identify and resolve these conflicts usually vary from one type of system to another. For example, the techniques we once proposed for database systems in [6] are quite different from the techniques we will propose for file systems in Section 4. However, we can generally classify these techniques into two categories: (1) Static Resolution allows both the main action history and the suspicious action histories to grow without any restrictions. 
Identification and resolution of conflicts are delayed until some suspicious history is designated to be merged into the main history. (2) Dynamic Resolution does not allow either the main history or the suspicious histories to grow unless the mutual consistency is guaranteed.

Finally, an isolation system should satisfy the following general requirements to ensure the security and correctness of intrusion confinement: (a) Disclosure Property: The isolation strategy should never cause risky data flows from suspicious data versions to the main data version. (b) Consistency Property: Before and after each merger, the main data version and the suspicious data versions should be kept consistent. The consistency of the main version can be relaxed when some suspicious updates are disclosed to it, and vice versa. (c) Synchronization Property: A suspicious data version cannot be merged into the main data version if it is still dependent on some other suspicious data versions.

\section{INTRUSION CONFINEMENT IN FILE SYSTEMS}

In this section, we will present a concrete isolation protocol in the file system context to evaluate the feasibility of our general intrusion confinement solution.

\subsection{ISOLATION PROTOCOL}

Consider a file system with a set of files, denoted $f_{1}, f_{2}, \ldots, f_{n}$, that are accessed by users. These files can be of many types, such as normal files, directories, and devices. A user can access a file in many ways, such as reading, writing, modifying, renaming, deleting, copying, and moving. In this section, we choose one-way isolation as the isolation strategy and complete merger as the merging strategy, and forbid data flows among suspicious versions.

The isolation protocol which is specified as follows is adapted from [16], where a protocol is proposed to detect and resolve mutual inconsistency in distributed file systems. In this protocol, the isolation is processed in terms of each file. When a file $f_{i}$ is modified by a suspicious user $S_{i}$, the modification and the possible following modifications of $S_{i}$ on $f_{i}$ will be isolated until $S_{i}$ proves to be malicious or innocent. To identify the conflicts between the modifications of $S_{i}$ on $f_{i}$ and the modifications of trustworthy users on $f_{i}$, we associate a version vector with the main version and every isolated version of $f_{i}$.

(a) Each file $f_{i}$ is associated with a system-wide, unique identifier, called origin point (denoted $O P\left(f_{i}\right)$ ), which is generated when $f_{i}$ is created. It is an immutable attribute of $f_{i}$, although $f_{i}$ 's name is not immutable (indeed, $f_{i}$ may have different names in different versions). Thus, no number of modifications or renamings of $f_{i}$ will change $O P\left(f_{i}\right)$. 
(b) At the very beginning when there are no suspicious users, the version vector of the main version of a file $f_{i}$ can be viewed as $\langle G: 0\rangle$, although we do not maintain such a vector until $f_{i}$ is updated by a suspicious user. Here, $G$ denotes the main version.

(c) When a file $f_{i}$ is firstly modified by a suspicious user (denoted $S_{1}$ ), first, an isolated version of $f_{i}$ (with the same $O P\left(f_{i}\right)$ ), called the $S_{1}$-version of $f_{i}$, is created for $S_{i}$. Second, $\left\langle G: 0, S_{1}: 0>\right.$ is created as the version vector for the main version. $S_{1}: 0$ signifies that the version has not been modified by $S_{1}$. $G: 0$ signifies that the version has not been modified by trustworthy users since $f_{i}$ is firstly modified by a suspicious user (here the user is $S_{1}$ ). Third, $<G: 0, S_{1}: m>$ is created as the version vector for the $S_{1}$-version. $S_{1}: m$ means that $f_{i}$ has been modified by $S_{1}$. Note that $m$ is not a number. The $S_{1}$ dimension remains to be $m$ no matter how many times the $S_{1}$-version is modified.

(d) The version vector of the main version of a file $f_{i}$, if it exists, is changed by modifications performed by trustworthy users as follows: (1) after each such modification, its value in the $G$ dimension is increased by 1 , i.e., from $G: n$ to $G: n+1$. (2) the value in any other dimension is unchanged.

(e) When a suspicious user $S_{i}$ asks to modify a file $f_{i}$ which has already been modified by some suspicious users, if a $S_{i}$-version of $f_{i}$ exists, then the version is given. Otherwise, we first insert into the main version vector of $f_{i}$ the $S_{i}$ dimension with the value $S_{i}: 0$, then create the $S_{i}$-version of $f_{i}$. The $S_{i}$-version vector is created by first copying the main version vector and then changing its value in the $S_{i}$ dimension to $S_{i}: m$.

(f) When a suspicious user $S_{i}$ asks to delete a file $f_{i}$, if there is an $S_{i}$-version of $f_{i}$, then (1) the $S_{i}$-version is deleted; (2) the value of the $S_{i}$-version vector in the $S_{i}$ dimension is changed to $S_{i}: d$, which indicates that the $S_{i}$-version is removed. However, the origin point $O P\left(f_{i}\right)$ associated with the $S_{i}$-version vector remains. Otherwise, the $S_{i}$ dimension with the value $S_{i}: 0$ is inserted into the main version vector, and the $S_{i}$-version vector is created by first copying the main version vector and then changing its value in the $S_{i}$ dimension to $S_{i}: d$.

(g) When a trustworthy user asks to delete a file $f_{i}$, if the main version exists, then the $G$ dimension of the main version vector (if any) is changed to $G: d$, and the main version will be removed. However, the origin point $O P\left(f_{i}\right)$ associated with the main version vector remains. We keep the vector because at this time some suspicious versions of $f_{i}$ could still exist.

(h) When a suspicious user $S_{i}$ (a trustworthy user) asks to access a file $f_{i}$ and the value of the $S_{i}$-version vector (the main version vector) in the $S_{i}(G)$ dimension is $d$, then the system notifies the user that $f_{i}$ does not exist. 
(i) When a suspicious $S_{i}$ asks to read a file $f_{i}$, if there is an $S_{i}$-version of $f_{i}$, then the version is given to $S_{i}$. Otherwise, if the main version exists, then it is given.

(j) When a suspicious version is merged with the main version, the main version vector of $f_{i}$ can have more dimensions than the suspicious version vector of $f_{i}$. To identify and resolve the conflicts between these two versions, we need to first pad the suspicious version vector such that the two vectors have the same set of dimensions. The padding is done by inserting each missed dimension with the value 0 into the suspicious version vector.

\subsection{RESOLUTION OF CONFLICTS}

There are two types of conflicts that we wish to consider: name conflicts and version conflicts. A name conflict occurs when two files with different origin points have the same name. In contrast, a version conflict occurs when two versions of the same file (the same origin point) have been incompatibly modified. Two versions of a file are compatible iff there is no version conflict between them.

When a suspicious data version is merged into the main data version, we identify the version conflicts on a file $f_{i}$ as follows: (a) If both the suspicious version of $f_{i}$ and the main version of $f_{i}$ are deleted, or at least one of them is deleted, then they are compatible. (b) If none of them are deleted, then the two versions are compatible if their corresponding version vectors are compatible. Two version vectors are compatible if one vector $\vec{v}_{i}$ dominates the other vector $\overrightarrow{v_{j}}$ in the value of every dimension. If so, we say $\overrightarrow{v_{i}}$ dominates $\vec{v}_{j}$. In our model, value domination is defined as follows: (1) A value dominates itself. (2) A number $n_{1}$ dominates another number $n_{2}$ if $n_{1}$ is larger than $n_{2}$. (3) The value $m$ (in $S_{i}: m$ ) dominates the value 0 . (4) The value $d$ is dominated by any other values.

Name conflicts can be easily resolved by renaming files after all of the version conflicts are resolved. Resolution of version conflicts should be accompanied by resolution of version vector conflicts because we cannot merge two versions without merging their version vectors. When the conflicts between a suspicious $S_{i}$-version (with version vector $\overrightarrow{v_{s}}$ ) and a main data version (with version vector $\overrightarrow{v_{g}}$ ) of a file $f_{i}$ are identified, we resolve the conflicts and merge the two versions as follows. The protocol can ensure that in the merged vector: (1) $G: d$ indicates the merged version is removed; otherwise, it exists. (2) $S_{i}: 0$ indicates there is (or was) a suspicious $S_{i}$-version. The version may still be active or may have already been discarded. (3) $S_{i}: d$ indicates there was a suspicious $S_{i}$-version that has been deleted by $S_{i}$, who is innocent. (4) $S_{i}: m$ indicates there was a suspicious $S_{i}$-version. The version is 
modified by $S_{i}$ and is merged.

(a) If both of the two versions are deleted, then they need not be merged; however, their version vectors need to be merged. The merging is done by taking $\overrightarrow{v_{g}}$ with its value in the $S_{i}$ dimension changed to $S_{i}: d$ as the merged vector.

(b) Suppose that the main version is deleted, but the $S_{i}$-version is not deleted. Then the $S_{i}$ version is the merged version, and the merged vector is $\overrightarrow{v_{g}}$ with its value in the $G$ dimension changed to that of $\overrightarrow{v_{s}}$ and its value in the $S_{i}$ dimension changed to $m$.

(c) Suppose that the $S_{i}$-version is deleted, but the main version is not deleted. If the value of $\overrightarrow{v_{g}}$ in the $G$ dimension is larger than that of $\overrightarrow{v_{s}}$, or there is a dimension such that the value of $\overrightarrow{v_{g}}$ in the dimension is $m$ but the value of $\overrightarrow{v_{s}}$ in the dimension is 0 or $\overrightarrow{v_{s}}$ does not include the dimension, then the main version is the merged version, and $\overrightarrow{v_{g}}$ with its value in the $S_{i}$ dimension changed to $S_{i}: d$ is the merged vector. Otherwise, the deleted $S_{i}$ version is the merged version, and $\overrightarrow{v_{g}}$ with its value in the $G$ dimension changed to $d$ and its value in the $S_{i}$ dimension changed to $d$ is the merged vector.

(d) If none of the two versions are deleted: (1) If $\overrightarrow{v_{g}}$ dominates $\overrightarrow{v_{s}}$, then the main version is the resolved version. $\overrightarrow{v_{g}}$ is the merged vector. (2) If $\overrightarrow{v_{s}}$ dominates $\overrightarrow{v_{g}}$, then the suspicious version is the resolved version. $\overrightarrow{v_{s}}$ is the merged vector. (3) If $\overrightarrow{v_{g}}$ and $\overrightarrow{v_{s}}$ are incompatible, then the resolution can be done either manually or automatically, based on the semantics of the modifications that have been applied on these versions. After the resolution, $\overrightarrow{v_{g}}$ with its value in the $S_{i}$ dimension changed to $m$ is the merged vector.

Example 2 Assume that when the $S_{2}$-version is merged into the main version, the main version vector of a file $f_{i}$ is: $\overrightarrow{v_{g}}\left(f_{i}\right)=<G: 0, S_{1}: 0, S_{2}: 0>$. At this point, if the $S_{2}$-version vector of $f_{i}$ is $\overrightarrow{v_{s}}\left(f_{i}\right)=<G: 0, S_{1}: 0, S_{2}: m>$, then since $\overrightarrow{v_{s}}\left(f_{i}\right)$ dominates $\overrightarrow{v_{g}}\left(f_{i}\right)$, so there are no conflicts, and the $S_{2}$-version is the merged version, and $\overrightarrow{v_{s}}\left(f_{i}\right)$ is the merged vector. If $\overrightarrow{v_{s}}\left(f_{i}\right)=<G: 0, S_{1}$ : $0, S_{2}: d>$, then the two versions are still compatible. The deleted $S_{2}$-version is the merged version, and $\left\langle G: d, S_{1}: 0, S_{2}: d>\right.$ is the merged vector.

Consider another scenario where $\overrightarrow{v_{g}}\left(f_{i}\right)=<G: 2, S_{1}: 0, S_{2}: 0>$ and $\overrightarrow{v_{s}}\left(f_{i}\right)=<G: 0, S_{1}: 0, S_{2}: m>. \overrightarrow{v_{g}}\left(f_{i}\right)$ and $\overrightarrow{v_{s}}\left(f_{i}\right)$ conflict. Note that the version of $f_{i}$ with the vector $\left\langle G: 0, S_{1}: 0, S_{2}: 0>\right.$ has been independently modified by $S_{2}$ and some trustworthy transactions. At this point, manual or automatic approaches need be applied to resolve the conflicts. The version vector of the resolved version is $\left\langle G: 2, S_{1}: 0, S_{2}: m\right\rangle$.

To reduce the overhead of maintaining version vectors, we need to reset main version vectors. The reset for the main version vector of $f_{i}$ can be processed when there are no active accesses to $f_{i}$ and when $f_{i}$ has not been modi- 
fied or deleted in any active suspicious versions. The reset can be easily done by removing the main version vector.

Although there is no general way to resolve conflicts automatically, conflict resolution can be automated in many file systems by exploiting the operation semantics. For example, reconciliation for two important types of files in LOCUS [16], directories and user mailboxes, is handled automatically. Interested readers can refer to [16] for more details on this topic.

\section{CONCLUSION}

In this paper, we propose, model, and analyze the problem of intrusion confinement, and we also illustrate how to solve it. It is shown that intrusion confinement can effectively resolve the conflicting design goals of an intrusion detection system by achieving both a high rate of detection and a low rate of errors. It is also shown that as a second level of protection in addition to access control intrusion confinement can dramatically enhance the security (especially integrity and availability) of a system in many situations. Although a concrete isolation scheme is proposed only for file systems, intrusion confinement can be enforced in many other types of information systems, such as database systems (a simple isolation scheme is proposed in [6]), objectoriented systems, distributed information systems, and workflow management systems. Developing concrete isolation protocols for these systems is a topic of our future research.

\section{Acknowledgments}

Jajodia and McCollum were partially supported by Rome Laboratory, Air Force Material Command, USAF, under agreement number F30602-97-1-0139.

\section{References}

[1] Ammann, P., Jajodia, S. and Liu, P. (1998). Recovery from malicious transactions. Technical report, George Mason University, Fairfax, VA. http://www.isse.gmu.edu/ pliu/papers/dynamic.ps.

[2] Ammann, P., Jajodia, S., McCollum, C. and Blaustein, B. (1997). Surviving information warfare attacks on databases. Proceedings of the IEEE Symposium on Security and Privacy, pp. 164-174, Oakland, CA.

[3] Graubart, R., Schlipper, L. and McCollum, C. (1996). Defending database management systems against information warfare attacks. Technical report, The MITRE Corporation.

[4] Ilgun, K. (1993). Ustat: A real-time intrusion detection system for unix. Proceedings of the IEEE Symposium on Security and Privacy. 
[5] Ilgun, K., Kemmerer, R. and Porras, P. (1995). State transition analysis: A rule-based intrusion detection approach. IEEE Transactions on Software Engineering, 21(3), pp. 181-199.

[6] Jajodia, S., Liu, P. and McCollum, C. (1998). Application-level isolation to cope with malicious database users. Proceedings of the Fourteenth Annual Computer Security Application Conference, pp. 73-82, Phoenix, AZ.

[7] Javitz, H. S. and Valdes, A. (1994). The nides statistical component description and justification. Technical Report A010, SRI International.

[8] Lin, J., Wang, X. S. and Jajodia, S. (1998). Abstraction-based misuse detection: High-level specifications and adaptable strategies. Proceedings of the Eleventh IEEE Computer Security Foundations Workshop.

[9] Liu, P., Ammann, P. and Jajodia, S. (2000). Rewriting histories: Recovery from malicious transactions. Distributed and Parallel Databases. To appear. http://www.isse.gmu.edu/ pliu/papers/semrepair.ps.

[10] Lunt, T. (1993). A survey of intrusion detection techniques. Computers \& Security, 12(4), pp. 405-418.

[11] Lunt, T. and McCollum, C. (1998). Intrusion detection and response research at DARPA. Technical report, The MITRE Corporation, McLean, VA.

[12] McDermott, J. and Goldschlag, D. (1996). Storage jamming. Database Security IX: Status and Prospects (eds. D. Spooner, S. Demurjian and J. Dobson), Chapman \& Hall, pp. 365-381.

[13] McDermott, J. and Goldschlag, D. (1996). Towards a model of storage jamming. Proceedings of the IEEE Computer Security Foundations Workshop, pp. 176-185.

[14] Mukherjee, B., Heberlein, L. T. and Levitt, K. (1994). Network intrusion detection. IEEE Network, pp. 26-41.

[15] Panda, B. and Giordano, J. (1999). Reconstructing the database after electronic attacks. Database Security XII: Status and Prospects (ed. S. Jajodia). Kluwer.

[16] Parker, D., Popek, G., Rudisin, G., Stoughton, A., Walker, B., Walton, E., Chow, J., Edwards, D., Kiser, S. and Kline, C. (1983). Detection of mutual inconsistency in distributed systems. IEEE Transactions on Software Engineering, 9(3), pp. 240-247.

[17] Porras, P. and Kemmerer, R. (1992). Penetration state transition analysis: A rule-based intrusion detection approach. Proceedings of the Eighth Annual Computer Security Applications Conference.

[18] Shieh, S.-P. and Gligor, V. (1991). A pattern oriented intrusion detection model and its applications. Proceedings of the IEEE Symposium on Security and Privacy.

[19] Shieh, S.-P. and Gligor, V. (1997). On a pattern-oriented model for intrusion detection. IEEE Transactions on Knowledge and Data Engineering, 9(4), pp. 661-667. 